\title{
K3 Rate Constant
}

National Cancer Institute

\section{Source}

National Cancer Institute. K3 Rate Constant. NCI Thesaurus. Code C80381.

A rate constant used in a three compartment tracer uptake model to represent the transport from a tissue non-metabolized compartment to a metabolized state. 